Math. Model. Nat. Phenom.

Vol. 5, No. 6, 2010, pp. 22-37

DOI: $10.1051 / \mathrm{mmnp} / 20105602$

\title{
Modelling the Spread of Infectious Diseases in Complex Metapopulations
}

\author{
J. Saldaña * \\ Departament d'Informàtica i Matemàtica Aplicada \\ Universitat de Girona, 17071 Girona, Catalonia, Spain
}

\begin{abstract}
Two main approaches have been considered for modelling the dynamics of the SIS model on complex metapopulations, i.e, networks of populations connected by migratory flows whose configurations are described in terms of the connectivity distribution of nodes (patches) and the conditional probabilities of connections among classes of nodes sharing the same degree. In the first approach migration and transmission/recovery process alternate sequentially, and, in the second one, both processes occur simultaneously. Here we follow the second approach and give a necessary and sufficient condition for the instability of the disease-free equilibrium in generic networks under the assumption of limited (or frequency-dependent) transmission. Moreover, for uncorrelated networks and under the assumption of non-limited (or density-dependent) transmission, we give a bounding interval for the dominant eigenvalue of the Jacobian matrix of the model equations around the disease-free equilibrium. Finally, for this latter case, we study numerically the prevalence of the infection across the metapopulation as a function of the patch connectivity.
\end{abstract}

Key words: metapopulations, infectious diseases, reaction-diffusion processes

AMS subject classification: 92D30, 92D40

\section{Introduction}

Multi-patch models for the spread of infectious diseases involving fluxes of individuals (migrants) among local populations have been considered in several papers (see, for instance, [10, 14, 17, $18,20])$. In most of the cases, when a general spatial arrangement of the patches is considered,

*E-mail: joan.saldana@udg.edu 
the graph describing it is assumed to be irreducible, i.e., the set of patches cannot be split in two groups such that there is no migration from one of the groups to the other one (see, for instance, $[9,20,13])$. This implies that the matrices $\left(a_{i j}\right)$ and $\left(b_{i j}\right)$ containing the non-negative migration rates $a_{i j}$ and $b_{i j}$ from patch $j$ to patch $i(i \neq j)$ of susceptible and infected individuals, respectively, must be irreducible (which guarantees the existence of a strictly dominant eigenvalue). Moreover, they must satisfy the balance condition given by

$$
\sum_{i} a_{i j}=\sum_{i} b_{i j}=0, \quad \forall j
$$

with $a_{i i} \leq 0, b_{i i} \leq 0$, and, for $i \neq j, a_{i j} \geq 0, b_{i j} \geq 0$. Other aspects of the metapopulation architecture as well as their influence on the epidemic dynamics have not been traditionally considered.

An alternative approach based on the formalism used in the statistical mechanics of complex networks is presented in [5, 7]. In these works, the architecture of the network of patches (nodes) where local populations live is mathematically encoded by means of the connectivity (degree) distribution $p(k)$, defined as the probability that a randomly chosen patch has degree $k$. As before, each patch contains both types of individuals occurring in the SIS model: susceptible and infected. Within each patch, transmission and recovery processes (reaction) occur at rates $\beta_{k}$ and $\mu$, respectively. Moreover, migratory flows (diffusion) take place among patches at emigration rates $D_{S}$ and $D_{I}$ for susceptible and infected individuals, respectively. Each diffusing individual randomly chooses one of the links departing from the patch. Therefore, the approach does not encompass a detailed description of the spatial network. Instead, it offers a description of the epidemic spread in terms of densities of susceptible and infected individuals in patches of degree $k$ at time $t$, here denoted by $\rho_{S, k}(t)$ and $\rho_{I, k}(t)$, respectively. Inside each patch, the transmission of the infection follows the SIS dynamics under the assumption of a homogenous mixing.

In the simplest version of this approach, which will be the one considered in this paper, emigration rates $D_{S}$ and $D_{I}$ are taken to be the same for all patches in the metapopulation [5, 19]. More elaborated models assume more generic types of migration where emigration rates are functions of the degree of the patches at both ends of a connection. Examples of this situation are given by the so-called traffic-dependent mobility rates where individuals' movements depend on the traffic intensity of connections, and by the population-dependent mobility rates, where rates are assumed to depend on the local population size in each patch (see [7] for details).

\section{The model equations}

\subsection{Discrete-time equations}

From the rates of recovery $\mu$ and transmission $\beta_{k}$, the probabilities of recovery and transmission during a small enough time interval $(t, t+\tau)$ are $\tau \mu$ and $\tau \beta_{k}$, respectively. Similarly, if migration (diffusion) can occur at any moment during this time interval, the migration probabilities of susceptible and infected individuals are, respectively, $\tau D_{S}$ and $\tau D_{I}$. According to this assumption 
and neglecting births and deaths (i.e., the total population remains constant at the metapopulation level), the discrete-time equations for migration and transmission/recovery processes read:

$$
\begin{aligned}
\rho_{S, k}(t+\tau)= & \left(1-\tau D_{S}\right)\left(\rho_{S, k}(t)+\tau \mu \rho_{I, k}(t)-\tau \beta_{k} \rho_{S, k}(t) \rho_{I, k}(t)\right) \\
& +k \tau D_{S} \sum_{k^{\prime}}\left(\rho_{S, k^{\prime}}(t)+\tau \mu \rho_{I, k^{\prime}}(t)-\tau \beta_{k^{\prime}} \rho_{S, k^{\prime}}(t) \rho_{I, k^{\prime}}(t)\right) P\left(k^{\prime} \mid k\right) \frac{1}{k^{\prime}}, \\
\rho_{I, k}(t+\tau)= & \left(1-\tau D_{I}\right)\left(\rho_{I, k}(t)+\tau \beta_{k} \rho_{S, k}(t) \rho_{I, k}(t)-\tau \mu \rho_{I, k}(t)\right) \\
& +k \tau D_{I} \sum_{k^{\prime}}\left(\rho_{I, k^{\prime}}(t)+\tau \beta_{k^{\prime}} \rho_{S, k^{\prime}}(t) \rho_{I, k^{\prime}}(t)-\tau \mu \rho_{I, k^{\prime}}(t)\right) P\left(k^{\prime} \mid k\right) \frac{1}{k^{\prime}},
\end{aligned}
$$

where $P\left(k^{\prime} \mid k\right)$ is the conditional probability that a patch (node) of degree $k$ has a connection (link) pointing to a patch of degree $k^{\prime}$. Since migration rates among patches are constant, emigrants from a patch with connectivity $k^{\prime}$ choose at random a neighbouring location with probability $1 / k^{\prime}$. Note that the probabilities of more than one event happening to the same individual are of order $\tau^{2}$ and, for $\tau$ small enough, this means that they are negligible when comparing them to one-event probabilities.

However, if emigration from a patch of degree $k^{\prime}$ is assumed to occur just at the end of the time interval, then a fixed fraction $D_{i}$ of $i$-individuals must be randomly selected, at time $t+\tau$, to be moved to a neighbouring location chosen at random with probability $1 / k^{\prime}$. In this situation, the probabilities $\tau D_{i}$ in Eqs. (2.1)-(2.2) must be replaced with $D_{i}$. Under this modelling assumption, the probabilities of more than one event (for instance, recover and migrate) occurring to the same individual are only of first order in $\tau$ and, then, they will not be negligible when passing to the continuous limit equations by taking $\tau \rightarrow 0$. Such a discrete-time diffusion was assumed in $[5,7]$ and, as it was observed in [2], is the reason for the problems arising in the Monte Carlo simulations of similar reaction-diffusion models when these processes occur sequentially in time (see also [11]).

The expression of the transmission rate $\beta_{k}$ in Eqs. (2.1)-(2.2) depends on the assumption about the number of contacts per unit of time. If we assume a fully mixed population, the contact rate $c$ is equal to the total population size in patch $k$, i.e., $\rho_{k}=\rho_{S, k}+\rho_{I, k}$, and we talk about non-limited or density-dependent transmission [12]. In this case, the transmission term per unit of time is given by

$$
\beta_{0} c \rho_{S, k} \rho_{I, k} / \rho_{k}=\beta_{0} \rho_{S, k} \rho_{I, k},
$$

where $\beta_{0}$ is the transmission rate across a contact with an infected individual [12]. Comparing with the previous equations, it follows that $\beta_{k}=\beta_{0}$. On the contrary, when the number of contacts per unit of time is fixed, $c$ does not depend on the population size, and for $c=1$ the transmission term per unit of time becomes

$$
\beta_{0} c \rho_{S, k} \rho_{I, k} / \rho_{k}=\beta_{0} \rho_{S, k} \rho_{I, k} / \rho_{k}
$$

and, hence, $\beta_{k}=\beta_{0} / \rho_{k}$. In this case, we have a limited or frequency-dependent transmission of the disease [12]. 


\subsection{Continuous-time equations}

When reaction and diffusion processes take place simultaneously, the equations for the continuoustime dynamics of the spread in a metapopulation are obtained from Eqs. (2.1)-(2.2) by taking the limit of $\left(\rho_{j, k}(t+\tau)-\rho_{j, k}(t)\right) / \tau$ when $\tau \rightarrow 0$, which amounts to the limit equations [19]

$$
\begin{aligned}
\frac{d}{d t} \rho_{S, k}(t) & =\rho_{I, k}\left(\mu-\beta_{k} \rho_{S, k}\right)-D_{S} \rho_{S, k}+k D_{S} \sum_{k^{\prime}} P\left(k^{\prime} \mid k\right) \frac{1}{k^{\prime}} \rho_{S, k^{\prime}}, \\
\frac{d}{d t} \rho_{I, k}(t) & =\rho_{I, k}\left(\beta_{k} \rho_{S, k}-\mu\right)-D_{I} \rho_{I, k}+k D_{I} \sum_{k^{\prime}} P\left(k^{\prime} \mid k\right) \frac{1}{k^{\prime}} \rho_{I, k^{\prime}} .
\end{aligned}
$$

As in classical reaction-diffusion equations, Eqs. (2.3)-(2.4) express the time variation of susceptible and infected individuals as the sum of two independent contributions: reaction and diffusion. In particular, the diffusion term includes the outflow of individuals (emigrants) from patches of degree $k$ and the inflow of individuals (immigrants) from the nearest patches. Here, we would like to mention that similar equations can be derived from the general formulation introduced in [2] for modelling reaction-diffusion processes of several types of particles in the so-called (in statistical mechanics) bosonic systems.

Notice that, since births and deaths are not considered in the model, the total number of individuals must be conserved at the metapopulation level. Precisely, after multiplying equations (2.3) and (2.4) by $p(k)$, and summing over all $k$, we have the following differential equations for $\rho_{S}(t)$ and $\rho_{I}(t)$, the average number of susceptible and infected individuals per patch at time $t$, respectively:

$$
\begin{aligned}
\frac{d}{d t} \rho_{S}(t) & =\sum_{k} p(k) \rho_{I, k}\left(\mu-\beta_{k} \rho_{S, k}\right)-D_{S} \rho_{S}+D_{S} \sum_{k} \sum_{k^{\prime}} k p(k) P\left(k^{\prime} \mid k\right) \frac{1}{k^{\prime}} \rho_{S, k^{\prime}} \\
\frac{d}{d t} \rho_{I}(t) & =\sum_{k} p(k) \rho_{I, k}\left(\beta_{k} \rho_{S, k}-\mu\right)-D_{I} \rho_{I}+D_{I} \sum_{k} \sum_{k^{\prime}} k p(k) P\left(k^{\prime} \mid k\right) \frac{1}{k^{\prime}} \rho_{I, k^{\prime}}
\end{aligned}
$$

with $\rho_{j}(t)=\sum_{k} p(k) \rho_{j, k}(t)(j=S, I)$. Now, since the number of links emanating from nodes of degree $k$ to nodes of degree $k^{\prime}$ must be equal to the number of links emanating from nodes of degree $k^{\prime}$ to nodes of degree $k$ in non-directed graphs, we have the following relationship between $p(k)$ and $P\left(k^{\prime} \mid k\right)$ (see [4])

$$
k P\left(k^{\prime} \mid k\right) p(k)=k^{\prime} P\left(k \mid k^{\prime}\right) p\left(k^{\prime}\right) .
$$

Using this restriction and the fact that $\sum_{k} P\left(k \mid k^{\prime}\right)=1$ after changing the order of summations, the previous equations become

$$
\begin{aligned}
\frac{d}{d t} \rho_{S}(t) & =\sum_{k} p(k) \rho_{I, k}\left(\mu-\beta_{k} \rho_{S, k}\right), \\
\frac{d}{d t} \rho_{I}(t) & =\sum_{k} p(k) \rho_{I, k}\left(\beta_{k} \rho_{S, k}-\mu\right) .
\end{aligned}
$$


Therefore, as expected, it follows that $d\left[\rho_{S}(t)+\rho_{I}(t)\right] / d t=0$, i.e., the total density of individuals $\rho(t)=\rho_{S}(t)+\rho_{I}(t)$ remains constant and equal to $\rho^{0}$, the initial average number of individuals per patch in the metapopulation. Note that the previous computations show that a balance condition equivalent to condition (1.1) is also fulfilled under the present modelling approach.

\subsection{Equations in uncorrelated networks}

To obtain analytical results about the epidemic dynamics in metapopulations, it is sometimes convenient to assume a particular function for $P\left(k^{\prime} \mid k\right)$. The usual thing is to restrict ourselves to uncorrelated networks. In these networks, the degrees of the nodes at the ends of any given link are independent, that is, there is no degree-degree correlation between the connected nodes. In this case, it follows that $P\left(k^{\prime} \mid k\right)=k^{\prime} p\left(k^{\prime}\right) / \sum_{k} k p(k)$ which corresponds to the degree distribution of nodes (patches) that we arrive at by following a randomly chosen link [15].

After substituting the expression of $P\left(k^{\prime} \mid k\right)$ into equations (2.3)-(2.4), one obtains the following equations for the epidemic spread in metapopulations described by uncorrelated networks:

$$
\begin{aligned}
\frac{d}{d t} \rho_{S, k}(t) & =\rho_{I, k}\left(\mu-\beta_{k} \rho_{S, k}\right)-D_{S}\left(\rho_{S, k}-\frac{k}{\langle k\rangle} \rho_{S}\right), \\
\frac{d}{d t} \rho_{I, k}(t) & =\rho_{I, k}\left(\beta_{k} \rho_{S, k}-\mu\right)-D_{I}\left(\rho_{I, k}-\frac{k}{\langle k\rangle} \rho_{I}\right),
\end{aligned}
$$

where $\langle k\rangle=\sum_{k} k p(k)$ is the average network connectivity.

In this form, it becomes clearer that the diffusion term is simply given by the difference between the outflow of susceptible (infected) individuals in patches of connectivity $k, D_{S} \rho_{S, k}\left(D_{I} \rho_{I, k}\right)$, and the total inflow of susceptible (infected) individuals across all their $k$ connections, which is $k$ times the average flow of individuals across a connection in the network, $D_{S} \rho_{S} /\langle k\rangle\left(D_{I} \rho_{I} /\langle k\rangle\right)$. Note that this average flow across a connection does not depend on the degree $k$ of the considered patch because we are assuming that the architecture of the metapopulation is described by an uncorrelated network.

\section{Equilibria}

\subsection{The disease-free equilibrium in generic networks}

The equilibria of the model equations (2.3)-(2.4) are the solutions $\rho_{S, k}^{*}, \rho_{I, k}^{*}$ to the equations

$$
\begin{aligned}
& \rho_{I, k}^{*}\left(\mu-\beta_{k} \rho_{S, k}^{*}\right)=D_{S}\left(\rho_{S, k}^{*}-k \sum_{k^{\prime}} P\left(k^{\prime} \mid k\right) \frac{1}{k^{\prime}} \rho_{S, k^{\prime}}^{*}\right), \\
& \rho_{I, k}^{*}\left(\beta_{k} \rho_{S, k}^{*}-\mu\right)=D_{I}\left(\rho_{I, k}^{*}-k \sum_{k^{\prime}} P\left(k^{\prime} \mid k\right) \frac{1}{k^{\prime}} \rho_{I, k^{\prime}}^{*}\right) .
\end{aligned}
$$


For the analysis of the infection's spread, it is particularly relevant the so-called disease-free equilibrium. By definition, this is obtained by replacing $\rho_{I, k}^{*}=0$ in the previous equations, leading to an implicit expression for the density of susceptible individuals in patches with degree $k$ that can be written as

$$
\frac{1}{k} \rho_{S, k}^{*}=\sum_{k^{\prime}} P\left(k^{\prime} \mid k\right) \frac{1}{k^{\prime}} \rho_{S, k^{\prime}}^{*}
$$

Note that, for those degrees $k$ that are not present in the network, $P\left(k^{\prime} \mid k\right)=0 \forall k^{\prime}$, and, so, $\rho_{S, k}^{*}=0$ for these degrees. From now on in the paper, when talking about degrees or connectivities, we implicitly mean those degrees or connectivities that are actually present in the network (see the numerical example at the end of this section). Furthermore, the case where all patches have the same connectivity is excluded from our considerations because, under the present approach, the model equations reduce to those of a single-patch SIS model.

Remark 1. The expression (3.3) says that, at equilibrium and for every $k$, the number of individuals leaving patches of connectivity $k$ per unit of time across one of their connections, $\rho_{S, k}^{*} / k$, equals the average number of individuals arriving at these patches per connection and per unit of time from the rest of the metapopulation (including patches with the same connectivity).

For networks with a connectivity pattern defined by the set of conditional probabilities $P\left(k^{\prime} \mid k\right)$ we have the following result that generalizes the one presented in [19] for uncorrelated networks:

Theorem 2. Consider the connectivity matrix $\mathcal{C}$ whose elements are given by

$$
\mathcal{C}_{k k^{\prime}}=\frac{k}{k^{\prime}} P\left(k^{\prime} \mid k\right)
$$

Then, for any conditional probabilities $P\left(k^{\prime} \mid k\right)$, the disease-free equilibrium is given by

$$
\rho_{S, k}^{*}=\rho_{k}^{*}=\frac{k}{\langle k\rangle} \rho^{0}, \quad \rho_{I, k}^{*}=0, \quad \forall k .
$$

Moreover, if $\mathcal{C}$ is irreducible and the transmission is frequency dependent, then the disease-free equilibrium is the only equilibrium for $\beta_{0}<\mu$ and it is unstable if and only if $\beta_{0}>\mu$.

Proof. From (3.3) it follows that $\rho_{S}^{*}$ must be an eigenvector of $\mathcal{C}$ with associated eigenvalue $\lambda=1$. Moreover, since $\sum_{k^{\prime}} P\left(k^{\prime} \mid k\right)=1$ for any degree $k$ in the network, it is immediate to see that $\mathcal{C}$ has the vector $v$ with components $v_{k}=k$ as eigenvector with associated eigenvalue $\lambda=1$. Therefore, $\rho_{S, k}^{*}=\alpha k$. Now, imposing that $\sum_{k} p(k) \rho_{S, k}^{*}=\rho^{0}$ if $\rho_{I, k}^{*}=0 \forall k$, it immediately follows that $\alpha=\rho^{0} /\langle k\rangle$.

To see that the disease-free equilibrium is the unique equilibrium for $\beta_{0}<\mu$, we only must multiply both sides of Eq. (3.2) by $p(k)$ and sum over all $k$. Then, while using the relationship (2.5) we have already seen that the right-hand side of the resulting equation equals 0 for any $\rho_{I, k}^{*} \geq 0$, the corresponding left-hand side amounts to

$$
\sum_{k} p(k) \frac{\rho_{I, k}^{*}}{\rho_{k}^{*}}\left(\left(\beta_{0}-\mu\right) \rho_{k}^{*}-\beta_{0} \rho_{I, k}^{*}\right)<0
$$


under the assumptions $\beta_{0}<\mu$ and $\rho_{I, k}^{*}>0$ for some $k$. So, the only equilibrium solution when $\beta_{0}<\mu$ is $\rho_{I, k}^{*}=0 \forall k$, i.e., the disease-free equilibrium.

Now, linearizing the system (2.3)-(2.4) about the disease-free equilibrium $\rho_{D F}^{*}$ given by (3.4), one obtains that the Jacobian matrix is of the form

$$
J\left(\rho_{D F}^{*}\right)=\left(\begin{array}{cc}
A & C \\
0 & B
\end{array}\right)
$$

where $A, B, C$, and 0 are $n \times n$ matrices with 0 being the zero matrix and $n$ the number of degrees in the metapopulation. Therefore, the characteristic polynomial of $J\left(\rho_{D F}^{*}\right)$ factorizes as $p_{J}(\lambda)=p_{A}(\lambda) p_{B}(\lambda)$ and, hence, the eigenvalues of $J\left(\rho_{D F}^{*}\right)$ are those of matrix $A$ plus those of matrix $B$.

Let $f_{i, k}$ denote the right-hand side of Eqs. (2.3)-(2.4) with $i=S, I$. Then, in a frequencydependent transmission of the disease, $\beta_{k}=\beta_{0} / \rho_{k}^{*}$ and the elements of the matrix $A$ are of the form

$$
A_{k k^{\prime}}=\frac{\partial f_{S, k}}{\partial \rho_{S, k^{\prime}}}\left(\rho_{D F}^{*}\right)=-D_{S} \delta_{k k^{\prime}}+D_{S} \mathcal{C}_{k k^{\prime}}
$$

where $\delta_{k k^{\prime}}$ is the Kronecker delta. Similarly, the elements of the matrix $B$ in this type transmission are of the form

$$
B_{k k^{\prime}}=\frac{\partial f_{I, k}}{\partial \rho_{I, k^{\prime}}}\left(\rho_{D F}^{*}\right)=\left(\beta_{0}-\mu-D_{I}\right) \delta_{k k^{\prime}}+D_{I} \mathcal{C}_{k k^{\prime}}
$$

From these expressions, it follows that the spectra of $A$ and $B, \sigma(A)$ and $\sigma(B)$, are shifted from those of $D_{S} \mathcal{C}$ and $D_{I} \mathcal{C}$ by $-D_{S}$ and $\beta_{0}-\mu-D_{I}$, respectively. Moreover, by the Perron-Frobenius theory (see, for instance, [3], Theorem 1.4, p. 27), we know that $\lambda=1$ is the dominant eigenvalue of $\mathcal{C}$ thanks to the assumed irreducibility of $\mathcal{C}$ and the positivity of the eigenvector associated with $\lambda=1$. This implies that $\lambda=0$ and $\lambda=\beta_{0}-\mu$ are, respectively, the eigenvalues of $A$ and $B$ with the largest real part. Consequently, in frequency-dependent transmission, the disease-free equilibrium is unstable when $\beta_{0}>\mu$.

Remark 3. The matrix $B$ defines the linear dynamics of infected individuals around the diseasefree equilibrium. Therefore, a positive dominant eigenvalue of $B$ implies an increase of the number of infected individuals initially added to a resident population of susceptible individuals at equilibrium. The dominant eigenvalue of $A$, which gives the growth rate of susceptible individuals about the disease-free equilibrium, is always 0 for any value of the rates $\beta_{0}$ and $\mu$ because of the conservation of the total number of individuals in the metapopulation.

Remark 4. The elements of the connectivity matrix $\mathcal{C}, k P\left(k^{\prime} \mid k\right) 1 / k^{\prime}$, are the average number of individuals that patches of degree $k$ receive from neighbouring patches of degree $k^{\prime}$ assuming that one individual leaves each of these patches by choosing at random one of the $k^{\prime}$ connections.

On the other hand, in density-dependent (or non-limited) transmission, $\beta_{k}=\beta_{0}$ and the Jacobian matrix around the disease-free equilibrium $\rho_{D F}^{*}$ has the same block structure as the one given by (3.5) but now with

$$
A_{k k^{\prime}}=\frac{\partial f_{S, k}}{\partial \rho_{S, k^{\prime}}}\left(\rho_{D F}^{*}\right)=-D_{S} \delta_{k k^{\prime}}+D_{S} \mathcal{C}_{k k^{\prime}}
$$


and

$$
B_{k k^{\prime}}=\frac{\partial f_{I, k}}{\partial \rho_{I, k^{\prime}}}\left(\rho_{D F}^{*}\right)=\left(\beta_{0} \rho_{k}^{*}-\mu-D_{I}\right) \delta_{k k^{\prime}}+D_{I} \mathcal{C}_{k k^{\prime}},
$$

where $\rho_{k}^{*}=\rho_{S, k}^{*}$ at the disease-free equilibrium. Hence, the elements of $B$ are not longer constant but depend on the densities $\rho_{k}^{*}$ of the disease-free equilibrium. In particular, as some elements on the main diagonal can be negative depending on $\rho_{k}^{*}$ and $\mathcal{C} \geq 0, B$ is a quasipositive matrix, i.e., its off-diagonal elements are nonnegative $\left(B_{k k^{\prime}} \geq 0\right.$ if $\left.k \neq k^{\prime}\right)$.

The latter means that, to obtain some information about the (in)stability of this equilibrium, we cannot proceed as in the previous theorem but need to use the following lemma about the stability modulus $\alpha(M)=\max \{\operatorname{Re}(\lambda): \lambda \in \sigma(M)\}$ of an irreducible quasipositive matrix $M$ :

Lemma 5. [9] Let $M$ be an irreducible, quasipositive matrix. Then,

1. If there exists a vector $x>0$ such that $M x>\underline{\lambda} x$ then $\alpha(M)>\underline{\lambda}$,

2. If there exists a vector $x>0$ such that $M x<\bar{\lambda} x$ then $\alpha(M)<\bar{\lambda}$,

where $x<(>)$ y means $x_{i} \leq(\geq) y_{i}$ and $x \neq y$. When $M$ is irreducible and nonnegative, then the same inequalities hold replacing $\alpha(M)$ by the dominant eigenvalue of $M$.

This lemma is a consequence of the Perron-Frobenius theory applied to the irreducible matrix $M+c I$ which is nonnegative for large enough $c \in \mathbb{R}$, and it allows us to prove the following:

Theorem 6. Consider the connectivity matrix $\mathcal{C}$ given in Theorem 2 . If $\mathcal{C}$ is irreducible and the transmission is density-dependent, then the stability modulus $\alpha(J)$ of the Jacobian matrix at the disease-free equilibrium satisfies $\alpha(J)=\max \{0, \alpha(B)\}$ with

$$
\beta_{0} \frac{k_{\min }}{\langle k\rangle} \rho^{0}-\mu<\alpha(B)<\beta_{0} \frac{k_{\max }}{\langle k\rangle} \rho^{0}-\mu
$$

Proof. Since $B$ is an irreducible, nonnegative matrix, we only need to find positive vectors for which the previous lemma holds. A natural candidate to begin with is the eigenvector $v$ of $\mathcal{C}$ associated with $\lambda=1$. For this vector $\left(v_{k}=k, k_{\min } \leq k \leq k_{\max }\right)$ we have

$$
B v=\left(\begin{array}{c}
k_{\min } \beta_{0} \rho_{k_{\min }}^{*} \\
\left(k_{\min }+1\right) \beta_{0} \rho_{k_{\min }+1}^{*} \\
\vdots \\
k_{\max } \beta_{0} \rho_{k_{\max }}^{*}
\end{array}\right)-\mu v>\left(\frac{k_{\min }}{\langle k\rangle} \beta_{0} \rho^{0}-\mu\right) v=: \underline{\lambda} v
$$

with $\rho_{k}^{*}$ given by (3.4). Similarly, we have that $B v<\left(\frac{k_{\max }}{\langle k\rangle} \beta_{0} \rho^{0}-\mu\right) v=: \bar{\lambda} v$. So, the statement of theorem follows from the previous lemma taking $x=v$.

Therefore, in contrast to what happens with limited transmission, for any value of $\beta_{0}$ and $\mu$, an infection will spread to the whole metapopulation if the mean number of susceptible individuals per patch $\rho^{0}$ exceeds a critical size. In particular, we have the following sufficient condition that holds for any generic network: 
Corollary 7. In non-limited transmission, the disease-free equilibrium becomes unstable if the mean number of individuals per patch in the metapopulation is large enough to guarantee that

$$
\rho^{0}>\frac{\langle k\rangle}{k_{\min }} \frac{\mu}{\beta_{0}}
$$

This condition simply says that, if the number of individuals inhabiting those patches with the lowest connectivity, $\rho_{\min }^{*}=\rho^{0} k_{\min } /\langle k\rangle$, is large enough, then infection reaches all patches. Indeed, this result is the one we would expect if we recall that, in a non-limited transmission of an infectious disease, the per capita number of contacts per unit of time in a patch is equal to the total population in this patch.

This estimation of the so-called epidemic threshold [12] will be significantly improved in the next subsection under the assumption of a particular network architecture which will allow to obtain an expression of the connectivity matrix.

\subsection{Equilibria in uncorrelated networks}

If we want to obtain results about endemic equilibria of the model and, moreover, to be more precise about the conditions for the (in)stability of the disease-free equilibrium in non-limited transmission, we must make an assumption about the degree-degree correlation in the network [16]. The simplest choice is given by uncorrelated networks. In this case, the equilibria of Eqs. (2.6)-(2.7) are the solutions $\rho_{S, k}^{*}, \rho_{I, k}^{*}$ of the system

$$
\begin{aligned}
& \rho_{I, k}^{*}\left(\mu-\beta_{k} \rho_{S, k}^{*}\right)=D_{S}\left(\rho_{S, k}^{*}-\frac{k}{\langle k\rangle} \rho_{S}^{*}\right) \\
& \rho_{I, k}^{*}\left(\beta_{k} \rho_{S, k}^{*}-\mu\right)=D_{I}\left(\rho_{I, k}^{*}-\frac{k}{\langle k\rangle} \rho_{I}^{*}\right)
\end{aligned}
$$

where $\rho_{j}^{*}=\sum_{k} p(k) \rho_{j, k}^{*}$ is the average number of individuals of type $j(j=S, I)$ per patch at equilibrium.

From these equations, it immediately follows that the disease-free equilibrium is given by Eq. (3.4). Note that, as in generic networks, the local population size is proportional to the connectivity $k$ of the patch. This is an effect of the pure diffusion of individuals among patches that has been already observed in other metapopulation models (see [7]).

In uncorrelated networks, the elements of the connectivity matrix $\mathcal{C}$ are simply given by

$$
\mathcal{C}_{k k^{\prime}}=\frac{k p\left(k^{\prime}\right)}{\langle k\rangle}
$$

Since all the rows of $\mathcal{C}$ are proportional to the first one, if there are $n$ different degrees in the metapopulation then the eigenvalues of this matrix are $\lambda=0$, with algebraic multiplicity $n-1$, and $\lambda=1$ which is a simple eigenvalue. In other words, $\mathcal{C}$ is a rank-one matrix with $\lambda=1$ as strictly dominant eigenvalue. Using this fact, one can easily prove the following: 
Theorem 8. In density-dependent transmission and assuming uncorrelated networks, the largest eigenvalue $\lambda_{\max }$ of the Jacobian matrix of Eqs. (2.6)-(2.7) around the disease-free equilibrium satisfies $\lambda_{\max }=\max \left\{0, \lambda_{k_{\max }}\right\}$ with

$$
\beta_{0} \frac{k_{\max }}{\langle k\rangle} \rho^{0}-\left(D_{I}+\mu\right)<\lambda_{k_{\max }}<\beta_{0} \frac{k_{\max }}{\langle k\rangle} \rho^{0}-\mu .
$$

Proof. In density-dependent transmission the Jacobian matrix of the system (2.6)-(2.7) around de disease-free equilibrium (3.3) is the block matrix given by (3.5) with $A$ and $B$ given by (3.6) and (3.7), respectively. Hence, the elements of $B$ are not constant but depend on the disease-free equilibrium. However, since now $\mathcal{C}$ has rank one, one can look at the matrix $B$ as a rank-one perturbation of the diagonal matrix $\left(\beta_{0} \rho_{k}^{*}-\mu-D_{I}\right) \delta_{k k^{\prime}}$ and use a well-known interlacing theorem of eigenvalues for this type of matrix perturbations to obtain the lower bound for $\lambda_{k_{\max }}$ (see, for instance, Theorem 0 in [1]). Finally, the upper bound follows from Theorem 6 and the fact all the eigenvalues of the Jacobian are real and, hence, $\alpha(J)=\lambda_{\max }$.

This result improves the one presented in $[19,11]$ for the largest eigenvalue of the Jacobian matrix since, for $D_{I} \ll \mu$, it gives an accurate bounding interval for $\lambda_{k_{\max }}$ and makes the following sufficient condition for an infection to invade close to be necessary:

Corollary 9. [19] In density-dependent transmission, a sufficient condition for the disease-free equilibrium to become unstable in uncorrelated networks is given by

$$
\rho^{0} \geq \frac{\langle k\rangle}{k_{\max }} \frac{\left(D_{I}+\mu\right)}{\beta_{0}} .
$$

Remark 10. This condition clearly improves the one in Corollary 7 when networks have large maximum degrees $k_{\max }$. Indeed, for very large networks with a bounded average degree $\langle k\rangle$ but an unbounded $k_{\max }$ as their size increases, condition (3.11) implies a practical lack of an epidemic threshold in the spread of the infection.

On the other hand, when transmission is frequency-dependent $\left(\beta_{k}=\beta_{0} / \rho_{k}\right)$, an endemic equilibrium of the system is simply obtained by imposing that both sides of equations (3.8)-(3.9) are equal to zero. This amounts to

$$
\rho_{S, k}^{*}=\frac{\mu}{\beta_{0}} \rho_{k}^{*}, \quad \rho_{I, k}^{*}=\left(1-\frac{\mu}{\beta_{0}}\right) \rho_{k}^{*},
$$

with $\rho_{k}^{*}=k \rho^{0} /\langle k\rangle$ being the total population size at equilibrium in patches of connectivity $k$. According to these expressions, the mean densities of susceptible and infected individuals in the metapopulation are $\rho_{S}^{*}=\mu / \beta_{0} \rho^{0}$ and $\rho_{I}^{*}=\left(1-\mu / \beta_{0}\right) \rho^{0}$, respectively. So, it is clear that the condition for the existence of an endemic equilibrium in this type of transmission is $\beta_{0}>\mu$. From this fact and from Theorem 2, it follows

Theorem 11. Let us assume frequency-dependent transmission in uncorrelated networks. Moreover, let us take $\beta_{0}$ as a bifurcation parameter. Then, for $\beta_{0}<\mu$, the disease-free equilibrium is the unique equilibrium of Eqs. (2.6)-(2.7) and is asymptotically stable. For $\beta_{0}>\mu$, the diseasefree equilibrium becomes unstable and appears an endemic equilibrium given by (3.12) which bifurcates from the disease-free equilibrium at $\beta_{0}=\mu$. 
Remark 12. For $\beta_{0}>\mu$, the matrix $B$, which defines the linear dynamics of infected individuals around the disease-free equilibrium, has all its eigenvalues positive. This implies an increase in the number of infected individuals in every patch regardless of its connectivity.

In the particular case of equal migration rates, $D_{S}=D_{I}=D$, from Eqs. (3.8)-(3.9) it immediately follows that $\rho_{k}^{*}=k \rho^{0} /\langle k\rangle$. Hence, for any given $\rho_{I}>0$, the density of infected individuals at equilibrium in patches of connectivity $k$ in terms of $\rho_{I}$ is given by

$$
\rho_{I, k}^{*}=\frac{k \rho^{0}}{2\langle k\rangle \beta_{0}}\left(\beta_{0}-\mu-D+\sqrt{\left(\beta_{0}-\mu-D\right)^{2}+4 \frac{\beta_{0}}{\rho^{0}} D \rho_{I}}\right) .
$$

Multiplying both sides of this expression by $p(k)$ and summing over all $k$, it follows that any feasible $\rho_{I}$ at equilibrium must satisfy

$$
\rho_{I}\left(\frac{\beta_{0}}{\rho^{0}} \rho_{I}-\left(\beta_{0}-\mu\right)\right)=0 .
$$

Therefore, the only positive mean density of infected individuals at an endemic equilibrium is given by $\rho_{I}^{*}=\left(1-\mu / \beta_{0}\right) \rho^{0}$, i.e., the one corresponding to the equilibrium (3.12), which implies uniqueness of endemic equilibrium of the model with frequency-dependent transmission and equal migration rates.

Finally, notice that at the endemic equilibrium (3.12) there is a balance between the inflow and the outflow of individuals in each patch of the metapopulation. This is so because, at this equilibrium, both sides of Eqs. (3.8)-(3.9) are equal to 0 and, hence, the flow of each type of individuals through a connection is the same for all the patches regardless their connectivity (see Remark 1):

$$
\frac{1}{k} \rho_{S, k}^{*}=\frac{1}{\langle k\rangle} \rho_{S}^{*}, \quad \frac{1}{k} \rho_{I, k}^{*}=\frac{1}{\langle k\rangle} \rho_{I}^{*}, \quad \forall k .
$$

In particular, this implies a constant prevalence of the disease (average fraction of infected individuals in patches of degree $k$ ) across the metapopulation, namely, $1-\mu / \beta_{0}$. In density-dependent transmission, however, such a local balance of flows does not occur because it is not possible for both sides of the equilibrium equations to vanish at the same time. Therefore, the behaviour of the prevalence of the disease will be completely different.

\subsection{Numerical study of the infection prevalence in density-dependent trans- mission on uncorrelated networks}

In density-dependent transmission $\left(\beta_{k}=\beta_{0}\right)$, the expression of the endemic equilibrium is not explicit (see [11] for a detailed analysis of this equilibrium as well as conditions for its existence and uniqueness). So, in order to see the behaviour of the prevalence of the infection in this type of transmission, we obtained a power-law degree distribution $p(k)$, with exponent $\gamma=3$ and average degree was $\langle k\rangle=6$, from a network of size $N=5000$ patches, which was used to integrate Eqs. (2.6)-(2.7) numerically. The generated network was uncorrelated (assortativity coefficient 
$r=-0.005$, see [16] for a definition), its minimum degree was $k_{\min }=3$, its maximum degree was $k_{\max }=232$, and there were $n=63$ different degrees.

From the numerical integration of the model equations with $\beta=1.5, \mu=5$, and $D_{S}=D_{I}=$ 1 , it follows that prevalence is an increasing function of the patch degree. Note that, since the migratory rates are equal, the total number of individuals in each patch is proportional to the patch degree. It is also observed a remarkable variation of the prevalence with the degree of the patch when the mean number $\rho^{0}$ of individuals per patch in the whole metapopulation is low. In Figure 1 , it is shown how prevalence varies with respect to patch connectivity for different $\rho^{0}$. For $\rho^{0}=6$, it ranges from 0.2149 for the smallest connectivities to 0.9843 for the largest ones. Increasing the average number of individuals, while maintaining constant the parameter values, causes an increase of the prevalence in all patches in the metapopulation and a significant reduction of its range of variation. For example, for $\rho^{0}=106$, the prevalence only varies from 0.9374 to 0.9992 . It is interesting to note that, for $\beta_{0}=1.5, \mu=5$, and the features of the network used in the simulations, an initial average density $\rho^{0} \geq 0.1034482$ will be enough to guarantee the instability of the disease-free equilibrium (see Corollary 9). The reason for such a low number of individuals per patch required for the invasion of the infection is the existence of a patch with a very high connectivity $\left(k_{\max }=232\right)$ that acts as a source of infectious individuals for the rest of the patches. Lower maximum degrees imply higher critical densities for the spread of the infection.

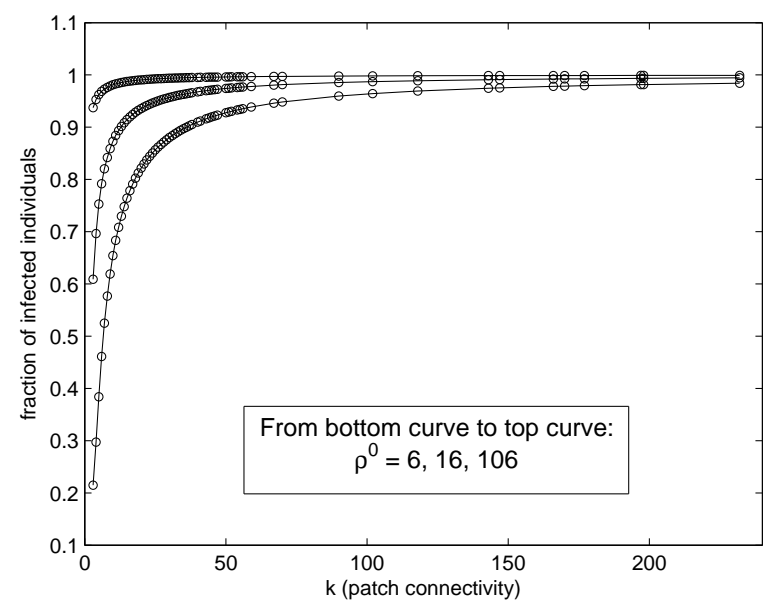

Figure 1: Prevalence of the infection in a metapopulation with a power-law degree distribution for different mean number of individuals per patch. Solutions are shown for $\rho^{0}$ equals 6 (lower curve), 16 (middle curve), and 106 (upper curve). Parameter values: $D_{I}=D_{S}=1, \beta=1.5, \mu=5$, $\langle k\rangle=6$. The empty dots correspond to connectivities present in the network while connecting lines are for eye guidance.

A similar behaviour of the solutions is obtained by varying the transmission rate across an infectious contact $\beta_{0}$. In Figure 2 we show the solutions for values of $\beta_{0}$ that are lower or equal to the recovery rate $\mu$. Note that, even for small values of the transmission rate $\left(\beta_{0}=0.1\right.$ and $\left.\mu=5\right)$, there exists an endemic equilibrium. Nevertheless, the profile of the prevalence for such a small 
value of $\beta_{0}$ is sigmoid shaped and, hence, different from the one obtained for larger values of $\beta_{0}$.

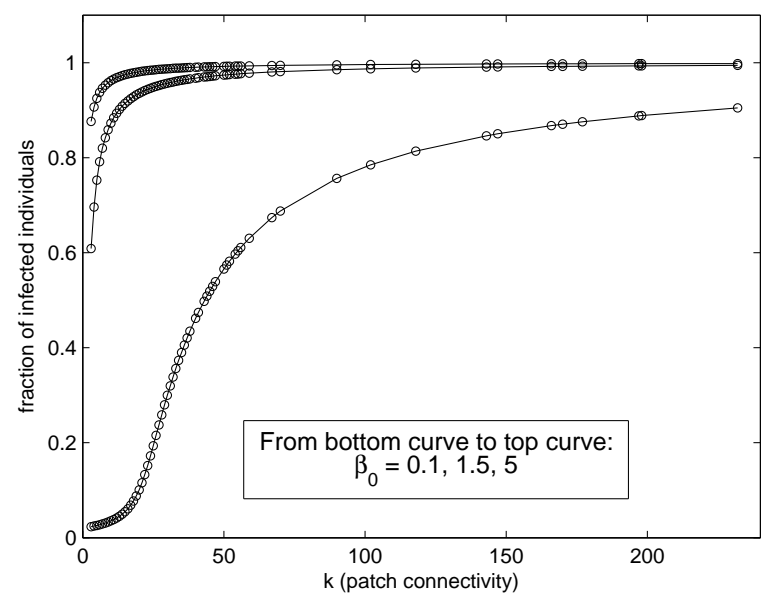

Figure 2: Prevalence of the infection in a metapopulation with a power-law degree distribution for different values of the transmission rate across an infectious contact. Solutions are shown for $\beta_{0}$ equals 0.1 (lower curve), 1.5 (middle curve), and 5 (upper curve). Parameter values: $D_{I}=D_{S}=1$, $\mu=5,\langle k\rangle=6, \rho^{0}=16$. The empty dots correspond to connectivities present in the network while the connecting lines are for eye guidance.

Finally, another interesting point is the role of $D_{I}$ in the prevalence of the infection. Diminishing the value of $D_{I}$ causes a reduction of the prevalence in patches of low connectivity, while those patches with higher connectivity have an even greater level of prevalence than the one occurring in the same patches with a higher $D_{I}$. In Figure 3, we show the prevalence for $\beta_{0}=1.5$, $\mu=5, \rho^{0}=16$ and two different values of $D_{I}$. For $D_{I}=0.1$, the prevalence goes from 0.1361 to 0.9977 and, for $D_{I}=2$, prevalence goes from 0.6401 to 0.9938 . This is the only situation we have observed in which the infection prevalence changes in a non-uniform manner across the metapopulation when changing the value of a parameter.

\section{Discussion}

This paper deals with a system of differential equations of reaction-diffusion type describing an epidemic spread in metapopulations based on the SIS model. The spatial configuration is given by the degree distribution $p(k)$ and the conditional probabilities $P\left(k^{\prime} \mid k\right)$. This formulation was derived in [19] and its main novelty with respect to previous models using the same approach was the assumption of simultaneous reaction and diffusion processes. Two limit cases for the disease transmission have been considered: a fixed number of contacts per unit of time (limited transmission) and a fully mixed population (non-limited transmission).

When a limited transmission is assumed, we have seen (Theorem 2) that the network architecture plays no role for the (in)stability of the disease-free equilibrium. Precisely, the epidemic 


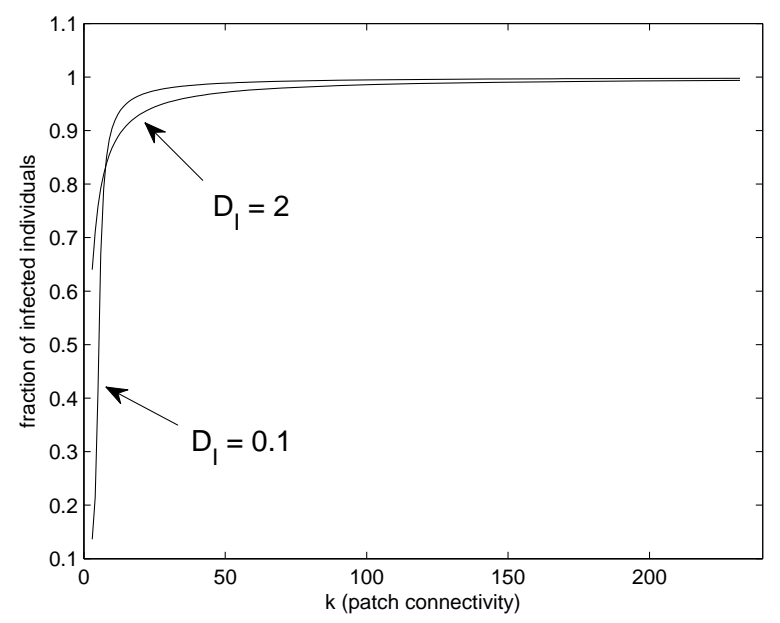

Figure 3: Prevalence of the infection in a metapopulation with a power-law degree distribution for two migration rates $D_{I}$ of infected individuals. Parameter values: $D_{S}=1, \beta=1.5, \mu=5$, $\langle k\rangle=6, \rho^{0}=16$. Only interpolating lines are drawn for visual distinction.

threshold at the metapolation level is the same as that of the SIS dynamics for a well-mixed populations living in a homogeneous environment, namely, $\beta_{0} / \mu>1$. In particular, this leads to a constant prevalence (fraction of infected individuals) of the infection across the metapopulation.

In a non-limited transmission and for generic networks, we have seen (Theorem 6) that the epidemics will progress at the whole metapopulation if it spreads in those nodes with the lowest connectivity. This is due to the fact that, in this type of transmission, the contact rate within a patch is assumed to be equal to the size of its population and, moreover, to migration among patches because it makes the (local) population size proportional to the patch connectivity. In the extreme case that all the patches have the same connectivity, i.e., a regular lattice, the bounding interval in the statement of Theorem 6 collapses $\left(k_{\min }=k_{\max }=\langle k\rangle\right)$ and leads to the condition for the instability of the disease-free equilibrium of the SIS model for well-mixed populations, namely, $\beta_{0} \rho^{0} / \mu>1$. Note that, under the present approach, all migratory flows are balanced when the spatial arrangement of the metapopulation is described by a regular lattice.

For a non-limited transmission in uncorrelated networks, a more precise bounding interval of the largest eigenvalue of the Jacobian matrix of the system around the disease-free equilibrium is presented in Theorem 8. From this interval, it follows the sufficient condition (3.11), which involves the migration rate of infected individuals and is close to be necessary for small values of this rate. This condition says that, for fixed $D_{I}, \mu$ and $\beta$, a high enough density of individuals and/or a large enough maximum connectivity in the metapopulation guarantee the instability of the disease-free equilibrium and, hence, the epidemic spread. In the limit of infinite networks with bounded average degree $\langle k\rangle$, this condition implies the lack of an epidemic threshold for any degree distribution with $k_{\max } \rightarrow \infty$. Additionally, the forecasted prevalence of the infection is not constant but increases with the patch connectivity (see the figures above). Interestingly, close the epidemic threshold, there are always patches with low connectivities where epidemics is not able 
to progress unless infected individuals arrive from (crowded) patches with higher connectivities.

The assumption of discrete-time diffusion ([5]) does not change the situation described above when a limited transmission is assumed. However, the scenario is completely different when transmission is non-limited. The main difference is with respect to the prevalence of the infection in the metapopulation because, in this case, the percentage of infected individuals is constant across the metapopulation.

All these models offer a deterministic description of the progress of a disease in a metapopulation and, so, they overlook the fact that extinction probabilities become relevant when a very low number of individuals invade non-infected patches. In this sense, the natural metric for the initial spread of a disease in a metapopulation is the number of local populations infected by individuals from an initially infected population, usually denoted by $R_{*}$ (see [8] for an interesting discussion and simulations). By assuming a statistical description of the metapopulation in terms of degree distributions, as in the present paper, the expression of an invasion threshold in uncorrelated networks is derived in [6] for discrete generations. The derivation of the threshold is based on the computation of $R_{*}$ for this type of networks and uses a branching process approximation for the time evolution of the total number of infected local populations in the $n$-th generation. However, in the SIS model and considering a well-mixed population, a certain fraction of infected individuals will be always present at equilibrium when the disease-free equilibrium is unstable. The latter implies that an epidemic outbreak in a given population will eventually reach other populations in the surrounding patches by repeated invasions. This fact somehow justifies neglecting the stochastic nature of invasion events and the use of a deterministic approach as the one adopted in this paper.

\section{Acknowledgements}

The author thanks David Juher and Jordi Ripoll for discussions on complex networks. The author also thanks David Juher for providing the network data for the numerical simulations. This work has been partially supported by the project MTM2008-06349-C03-02 of the Spanish government.

\section{References}

[1] J. Anderson. A secular equation for the eigenvalues of a diagonal matrix perturbation. Linear Algebra Appl. 246 (1996), 49-70

[2] A. Baronchelli, M. Catanzaro, R. Pastor-Satorras. Bosonic reaction-diffusion processes on scale-free networks. Phys. Rev. E 78 (2008), 016111

[3] A. Berman, R.J. Plemmons. Nonnegative matrices in the mathematical sciences. SIAM, Classics in Applied Mathematics 9, Philadelphia, PA, 1994.

[4] M. Boguñá, R. Pastor-Satorras. Epidemic spreading in correlated complex networks. Phys. Rev. E 66 (2002), 047104 
[5] V. Colizza, R. Pastor-Satorras, A. Vespignani. Reaction-diffusion processes and metapopulation models in heterogeneous networks. Nat. Phys. 3 (2007), 276-282

[6] V. Colizza, A. Vespignani. Invasion Threshold in Heterogeneous Metapopulation Networks. Phys. Rev. Lett. 99 (2007), 148701

[7] V. Colizza, A. Vespignani. Epidemic modeling in metapopulation systems with heterogeneous coupling pattern: Theory and simulations. J. theor. Biol. 251 (2008), 450-467

[8] P. C. Cross, P. L. F. Johnson, J. O. Lloyd-Smith, W. M. Getz. Utility of $R_{0}$ as a predictor of disease invasion in structured populations. J. R. Soc. Interface, 4 (2007), 315-324

[9] A. Fall, A. Iggidr, G. Sallet, J.J. Tewa. Epidemiological models and Lyapunov functions. Math. Model. Nat. Phenom. 2 (2007), 62-83

[10] L. Hufnagel, D. Brockmann, T. Geisel. Forecast and control of epidemics in a globalized world. PNAS 101 (2004), 15124-15129

[11] D. Juher, J. Ripoll, J. Saldaña. Analysis and Monte-Carlo simulations of a model for the spread of infectious diseases in heterogeneous metapopulations. Phys. Rev. E 80 (2009) 041920.

[12] M. J. Keeling, P. Rohani. Modeling infectious diseases in humans and animals. Princeton University Press, 2008.

[13] J. Li, X. Zou. Dynamics of an epidemic model with non-local infections for diseases with latency over a patchy environment. J. Math. Biol. (2009) DOI 10.1007/s00285-009-0280-9

[14] L. S. Liebovitch, I. B. Schwartz. Migration induced epidemics: dynamics of flux-based multipatch models. Phys. Lett. A 332 (2004), 256-267

[15] M. E. J. Newman, S. H. Strogatz, D. J. Watts. Random graphs with arbitrary degree distributions and their applications. Phys. Rev. E 64 (2001), 026118

[16] M. E. J. Newman. Mixing patterns in networks. Phys. Rev. E 67 (2003), 026126

[17] Y.-A. Rho, L. S. Liebovitch, I. B. Schwartz. Dynamical response of multi-patch, flux-based models to the input of infected people: Epidemic response to initiated events. Phys. Lett. A 372 (2008), 5017-5025

[18] L.A. Rvachev, I.M. Longini. A mathematical model for the global spread of influenza. Math. Biosci. 75 (1985), 3-22

[19] J. Saldaña. Continuous-time formulation of reaction-diffusion processes on heterogeneous metapopulations. Phys. Rev. E 78 (2008), 012902

[20] W. Wang, X.-Q. Zhao. An epidemic model in a patchy environment. Math. Biosci. 190 (2004), $97-112$ 